ISSN: 1576-0162

\title{
ANÁLISIS DEL GRADO DE EFICIENCIA DÉBIL EN ALGUNOS MERCADOS financieros europeos. Primer impacto del COVID-19

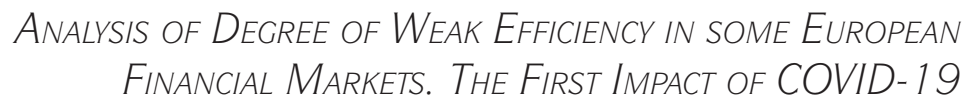 \\ $M^{a}$ B. García-Moreno García Universidad de Córdoba, Spain \\ José A. Roldán-Casas Universidad de Córdoba, Spain
}

Recibido: enero 2021; aceptado: junio 2021

\section{RESUMEN}

En este artículo se evalúa el grado de cumplimiento de la hipótesis de eficiencia débil en los mercados financieros de España, Alemania, Francia e Italia en el período 1 de enero de 2010 a 15 de mayo de 2020. Los resultados indican que los mercados analizados son eficientes en la forma que establece el paseo aleatorio 3 si bien el español es el que muestra menor grado de eficiencia. Los cuatro mercados presentan la mayor volatilidad del período a mediados de marzo de 2020, tras la declaraciōn de la pandemia del COVID-19 por la OMS.

Palabras clave: eficiencia, mercados financieros, paseo aleatorio, COVID-19.

\section{ABSTRACT}

In this paper is evaluated the degree of fulfilment of the hypothesis of weak efficiency in the financial markets of Spain, Germany, France and Italy, from $1^{\text {st }}$ January 2010 to $15^{\text {th }}$ May 2020. The results show that the markets are efficient in the form established by the random walk 3 . On the other hand, all the markets show their highest volatility in the middle of March 2020, just after the WHO declared the COVID-19 pandemic.

Keywords: Efficiency, financial markets, random walk, COVID-19.

Clasificación JEL / JEL Classification: G12, G14, C12. 



\section{INTRODUCCIÓN}

La hipótesis de eficiencia de los mercados financieros es una aproximaciōn analítica que surge con la pretensión de explicar los movimientos de las cotizaciones de los activos financieros a lo largo del tiempo, y pivota sobre la idea de que los precios de dichos activos vienen determinados por el comportamiento racional de los agentes que interactúan en el mercado. En este sentido, la hipótesis de eficiencia sustenta que los precios de los valores reflejan toda la información accesible a los agentes en el momento en que se determinan, por lo que, de cumplirse, no sería posible anticiparse a los cambios de precios y formular estrategias de inversión encaminadas a obtener cuantiosas rentabilidades. En otras palabras, no se podrían hacer predicciones acerca del comportamiento futuro del mercado.

El interés que tiene la verificación de la hipótesis de eficiencia en un mercado financiero, tanto para los inversores como para las instituciones que intervienen en la regulación de los mercados bursátiles, radica en que proporciona un elemento de juicio que permite evaluar si se están dando o no las condiciones que se requieren para que todos los agentes que actúan en un mercado lo hagan al amparo de lo que se conoce como "juego justo", esto es, en un escenario donde todos actúen en igualdad de condiciones, de manera que la expectativa de ganar sea igual a la de perder.

Por otro lado, puesto que la globalización del entorno económico y financiero conduce a la internacionalizaciōn de las finanzas, resulta evidente que los acontecimientos acaecidos en cualquiera de los principales mercados bursátiles, sean positivos o negativos, se ven reflejados en el resto de mercados a medida que se van abriendo las sesiones de los mismos. Ello ocasiona que los flujos de inversión extranjera sean trasladados de unos mercados a otros en función de factores como la rentabilidad, el riesgo o la seguridad, entre otros, que ofrezca un determinado mercado o país.

Se puede decir pues, que debido a la velocidad con la que pueden producirse las transacciones financieras, asi como a la interconexiōn que tienen unos mercados con otros, los escenarios que se presentan en el mundo de las finanzas son tan cambiantes y siempre orientados a estrategias para batir al 
mercado que, de cumplirse la hipótesis de mercado eficiente en cualquiera de ellos, debería hacerlo únicamente de forma transitoria. ${ }^{1}$

Aunque el origen teórico de la hipótesis de eficiencia se sitúa en 1900 con el trabajo de Bachelier, no es hasta 1965 cuando, por un lado, Samuelson fundamenta teóricamente dicha hipótesis, y por otro, Fama establece por primera vez el concepto de mercado eficiente. Poco tiempo después, Roberts (1967) acuña el término hipótesis de mercado eficiente en un trabajo en el que, además, analiza la eficiencia desde un punto de vista informacional, lo cual le lleva a establecer una clasificación para el conjunto de informaciōn que permite distinguir tres formas de eficiencia según el acceso creciente al mismo que tengan los agentes participantes en el mercado: débil, semi-fuerte y fuerte. Asī, en la eficiencia débil la informaciōn disponible para los agentes es únicamente la que constituye la serie histórica de precios; en la semi-fuerte el conjunto de información incluye toda la información pública disponible para todos los agentes; y en la fuerte el conjunto de información está constituido por la informaciōn de las dos formas anteriores y otra de carácter privado que se conoce como información privilegiada.

La forma débil de la hipótesis de eficiencia ha sido la protagonista indiscutible de gran parte de los estudios teóricos y empíricos que se han realizado a lo largo de la historia. En relación con las aportaciones teóricas, la gran mayoría de ellas identifican la hipótesis de eficiencia débil con el hecho de que el modelo de fijaciōn de precios de los activos financieros en el mercado objeto de análisis es el modelo de martingala o el denominado paseo aleatorio (en alguna sus formas, 1, 2 o 3). Ahora bien, puesto que para obtener hipótesis contrastables derivadas del modelo de martingala es necesario imponer restricciones adicionales sobre las distribuciones de probabilidad subyacentes que conducen a alguna de las versiones de paseo aleatorio, parece lógico asumir como modelo de fijación de precios únicamente alguna de sus formas.

En concreto, los tipos de paseo aleatorio con los que se identifica la hipótesis de eficiencia débil suponen condiciones que se imponen sobre los rendimientos asociados a los precios de un activo financiero, las cuales se van relajando desde el paseo aleatorio 1 (que es el que establece condiciones más estrictas) hasta el paseo aleatorio 3 (que se corresponde con el más plausible en términos económicos al no ser tan restrictivo), lo cual hace posible evaluar el grado de la eficiencia débil.

Pues bien, en relaciōn al estudio del cumplimiento de la hipótesis de la eficiencia financiera, el trabajo se plantea con los siguientes objetivos principales:

- Estudiar la evolución de algunos de los mercados financieros más representativos de Europa: España, Alemania, Francia e Italia.

\footnotetext{
${ }^{1}$ Con independencia de que la eficiencia se pueda considerar o no transitoria, es un hecho que los periodos de ineficiencia son más largos que los de eficiencia ya que el mercado es batido por los inversores constantemente. Esta realidad es cada vez más frecuente y habitual gracias a los avances de las nuevas tecnologías y de la minería de datos, que están muy orientados a la formación de estrategias real-time para la obtenciōn de ganancias (p.ej. trading de alta frecuencia hecho por programas informáticos bots).
} 
- Analizar el cumplimiento de la hipótesis de eficiencia en su forma débil en los mercados financieros objeto de estudio en el período 1 de enero de 2010 a 15 de mayo de 2020, prestando especial atención a la influencia que haya podido tener el inicio de la crisis del COVID-19².

- Proponer una forma de evaluar el grado de cumplimiento de la hipótesis de eficiencia débil a partir del procedimiento aplicado para contrastar dicha hipótesis.

- Analizar el grado de cumplimiento de la hipótesis de eficiencia débil en cada uno de los mercados estudiados.

Aunque existen numerosos procedimientos que tradicionalmente han sido utilizados para contrastar la eficiencia débil de un mercado financiero tal y como establece el modelo de paseo aleatorio, muchos de ellos contrastan únicamente alguna condición necesaria, pero no suficiente, del citado modelo en cualquiera de sus formas (es el caso, por ejemplo, de los llamados métodos lineales que solo contrastan la incorrelación necesaria para los tres tipos de paseo aleatorio) por lo que pueden llevar a una conclusión errónea.

Por tanto, en este trabajo el cumplimiento de la hipótesis de eficiencia débil se determina aplicando el procedimiento propuesto por García-Moreno (2015) en el que se combinan estratégicamente los métodos que contrastan directamente un tipo de paseo aleatorio y los que no, de tal manera que permite establecer si una serie de rendimientos financieros sigue o no un paseo aleatorio y, en su caso, de qué tipo se trata. Este procedimiento no tiene la limitaciōn, ya comentada, que presentan algunos métodos tradicionales al estudiar la hipótesis de eficiencia débil, relativa a que únicamente permiten contrastar alguna condición necesaria, pero no suficiente, para aceptar la hipótesis de paseo aleatorio en alguna de sus formas.

La evaluación del grado de cumplimiento de la hipótesis de eficiencia débil en cada mercado de acuerdo con alguno de los tres tipos de paseo aleatorio (1, 2 o 3), se realiza en base a una escala que asigna valores al resultado al que conduce el procedimiento de contraste señalado en el párrafo anterior tras ser aplicado a la serie de rendimientos correspondiente. En caso de rechazar la eficiencia, el valor asignado es el 0 , mientras que si se produce la aceptación de la hipótesis de paseo aleatorio el valor se asigna en función del mayor o menor nivel de exigencia de las condiciones asociadas al tipo de paseo del que se trate, es decir, a la aceptación de los tipos 1 y 3 se le asigna los valores 3 y 1 , respectivamente.

En consecuencia, el trabajo comienza (Secciōn 2) describiendo cómo se evalúa la hipótesis de eficiencia en un mercado financiero en base a la denominada hipótesis conjunta de Fama (1991). En la Sección 3 se detallan los diferentes métodos que tradicionalmente se aplican para contrastar la eficiencia débil en las formas que establecen los tipos de paseo aleatorio. Asimismo, se describe

2 Dado el carácter transitorio que suele tener el cumplimiento de la hipótesis de eficiencia financiera, el período muestral considerado finaliza el 15 de mayo de 2020, es decir, transcurridos, aproximadamente, dos meses desde la declaración de la pandemia del COVID-19 por parte de la OMS. 
el procedimiento que se aplica para detectar si una serie de rendimientos asociados a un determinado índice de precios sigue un paseo aleatorio y, en su caso, de qué tipo se trata. Finalmente, se propone una forma para evaluar el grado de cumplimiento de la hipótesis de eficiencia débil a partir del procedimiento aplicado. En la Secciōn 4 se concreta la metodología que se aplica para analizar la eficiencia débil en los mercados objeto de estudio, recogiéndose en la Sección 5 los resultados del citado análisis. Por último, la Sección 6 expone las principales conclusiones del trabajo.

\section{EVALUACIÓN DE LA HIPÓTESIS DE EFICIENCIA}

Para evaluar la eficiencia de un mercado financiero Bailey (2005) propone un procedimiento haciendo valer la idea de hipótesis conjunta de Fama (1991), esto es, considerando, además de la informaciōn disponible, la existencia de un modelo subyacente a la fijación de los precios de los activos financieros. En concreto, en base al citado modelo y al mencionado conjunto de información, se establece el criterio que determina la eficiencia del mercado que se traduce en una hipótesis contrastable. Así, mediante algún método o prueba diseñado para contrastar la hipótesis de eficiencia planteada se analiza si los datos recogidos (precios observados) evidencian o no dicha hipótesis, lo cual implicaría la eficiencia o ineficiencia del mercado, respectivamente. Todo este proceso queda recogido de forma esquemática en la Figura 1.

Es evidente que con el enfoque de este procedimiento la eficiencia de un mercado depende del modelo de fijación de precios y del conjunto de informaciōn asumidos. Esto significa que si la conclusiōn para un mercado es de eficiencia (ineficiencia) dados un modelo de fijaciōn de precios y un conjunto de información concretos, puede ocurrir que se concluya ineficiencia (eficiencia) si se asumen otro modelo y/o conjunto distintos.

Tradicionalmente la martingala y el paseo aleatorio son asumidos como modelos de fijación de precios $P_{t}$. de un activo financiero, cuyo rendimiento continuo o logarítmico viene dado por la expresiōn

$$
r_{t}=\ln P_{t}-\ln P_{t-1}=p_{t}-p_{t-1}
$$

Puesto que para obtener, en la práctica, hipótesis contrastables derivadas del modelo de martingala suelen imponerse restricciones adicionales sobre las distribuciones de probabilidad subyacentes, que dan como resultado las condiciones de alguna de las versiones de paseo aleatorio que Campbell et al. (1997) distinguen (Bailey, 2005, pp. 59-60), lo habitual es asumir como modelo de fijaciōn de precios el paseo aleatorio.

En sus inicios la formulación de paseo aleatorio fue

$$
p_{t}=p_{t-1}+r_{t}
$$


FIGURA 1: ESOUEMA DEL PROCEDIMIENTO PARA EVALUAR LA EFICIENCIA DE UN MERCADO

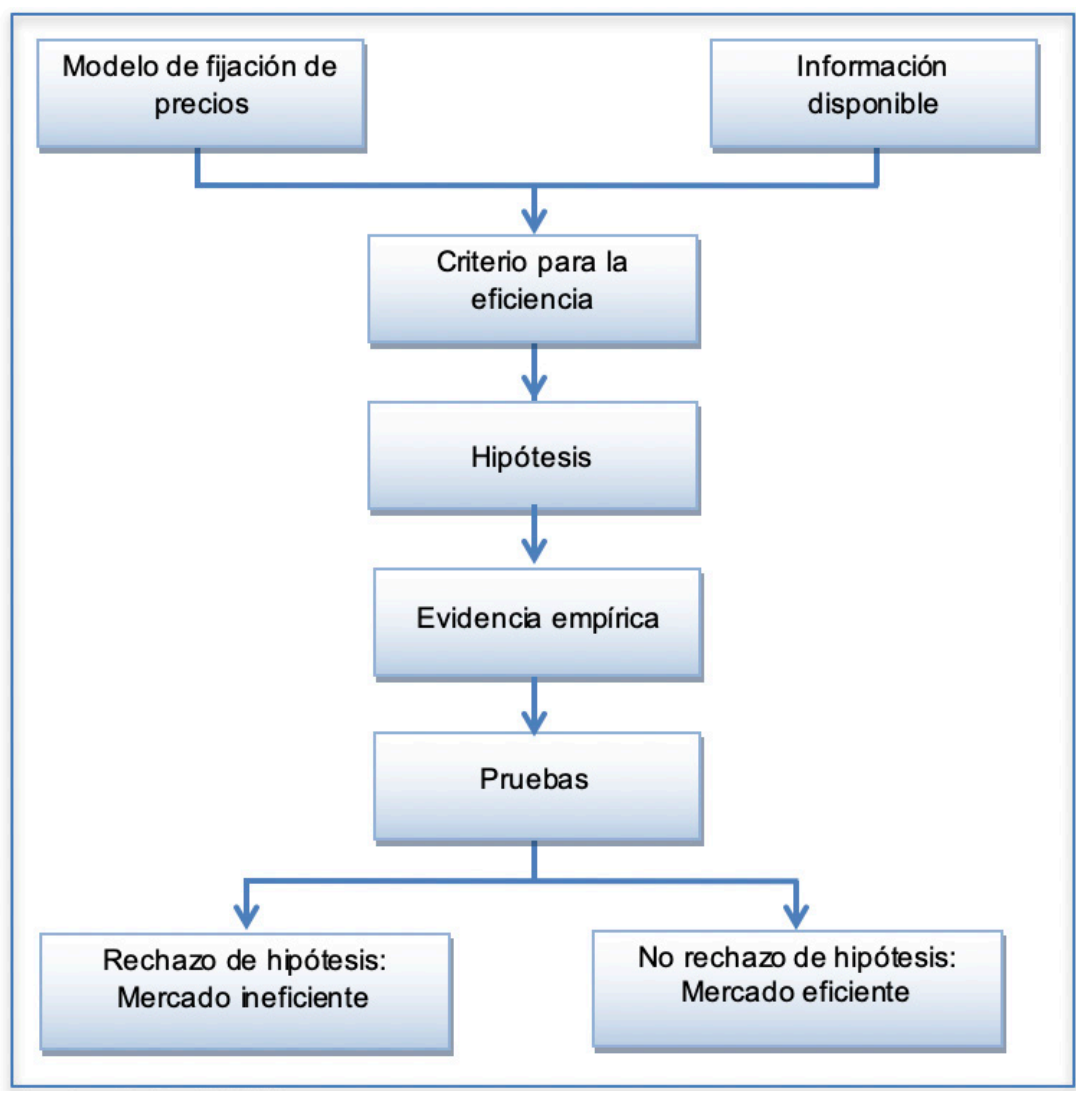

Fuente: Bailey (2005).

donde el término $r_{\text {t }}$ se consideraba un proceso independiente e idénticamente distribuido con media cero y varianza constante, lo cual supone que los cambios en los precios son impredecibles y aleatorios, hecho consustancial con las primeras versiones de la hipótesis de mercado eficiente. No obstante, diversos estudios de la época pusieron de manifiesto que lo datos financieros eran inconsistentes con estas condiciones.

Campbell et al. (1997) retomaron la idea de paseo aleatorio, pero en base a la formulaciōn

$$
p_{t}=\mu+p_{t-1}+\varepsilon_{t}
$$


donde $\mu$ es un término constante; y estableciendo condiciones sobre la estructura de dependencia del proceso $\left\{\varepsilon_{t}\right\}$ (que los autores denominan incrementos) distinguen tres tipos de paseo aleatorio: 1, 2 y 3. Ahora bien, como en este caso el cambio en el precio o rendimiento es

$$
p_{t}-p_{t-1}=r_{t}=\mu+\varepsilon_{t}
$$

las condiciones que se fijan sobre los incrementos $\left\{\varepsilon_{t}\right\}$ son extrapolables integramente a los rendimientos $\left\{\varepsilon_{t}\right\}$

\section{PASEO aleatorio 1 (RW1) 3 : INCREMENTOS/Rendimientos IID}

En este primer tipo, $\varepsilon_{\text {t }}$ es un proceso independiente e idénticamente distribuido con media 0 y varianza $\sigma^{2}$ o de forma abreviada $\varepsilon_{t} \sim \| D\left(0, \sigma^{2}\right)$, lo cual implica que $r_{t} \sim \| D\left(\mu, \sigma^{2}\right)$, de manera que la formulación (2) resulta ser un caso particular de este tipo de paseo aleatorio para $\mu=0$. Bajo estas condiciones el término constante $\mu$ queda como el cambio esperado en el precio o deriva.

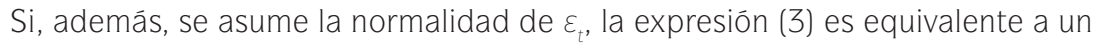
movimiento browniano aritmético.

En este caso, la independencia de $\varepsilon_{\text {t }}$ implica que el paseo aleatorio 1 es, además, un juego justo, pero en un sentido mucho más estricto que la martingala, ya que la citada independencia no solo implica que los incrementos/ rendimientos son incorrelados sino que cualesquiera funciones no lineales de los mismos son también incorreladas.

\section{PASEO ALEATORIO 2 (RW2): INCREMENTOS/RENDIMIENTOS INDEPENDIENTES}

Para este tipo de paseo aleatorio, $\varepsilon_{t}$, y por extensión $r_{t}$, es un proceso independiente pero no idénticamente distribuido (INID), estando contenido el RW1 como caso particular.

Esta versión del paseo aleatorio da cabida a procesos de generación de precios más generales y, al mismo tiempo, más acordes con la realidad del mercado pues, por ejemplo, permite heterocedasticidad no condicional en $r_{t}$, teniendo así en cuenta la dependencia temporal de la volatilidad característica de las series financieras.

\footnotetext{
${ }^{3}$ Se ha mantenido la denominaciōn original de los autores en la que las siglas RW corresponden a la expresión paseo aleatorio en inglés (random walk).
} 
PASEO ALEATORIO 3 (RW3): INCREMENTOS/RENDIMIENTOS INCORRELADOS

Bajo esta denominaciōn, $\varepsilon_{t}$, y por consiguiente $r_{t}$, es un proceso que no es independiente, ni está idénticamente distribuido, pero es incorrelado, es decir, se contemplan casos como

$$
\operatorname{Cov}\left(\varepsilon_{t}, \varepsilon_{t-k}\right)=0 \quad \forall k \neq 0 \text { pero } \operatorname{Cov}\left(\varepsilon_{t}^{2}, \varepsilon_{t-k}^{2}\right) \neq 0 \text { para algún } k \neq 0
$$

lo que quiere decir que puede haber dependencia, pero no correlación.

Esta es la versión más débil de la hipótesis de paseo aleatorio que contiene a los tipos 1 y 2 como casos especiales.

Como ya se ha comentado anteriormente, los datos financieros suelen rechazar el paseo aleatorio 1, principalmente, por incumplir el supuesto de constancia de la varianza de $r_{\text {t }}$. En cambio, los paseos aleatorios 2 y 3 se ajustan mejor a la realidad financiera pues permiten la existencia de heterocedasticidad (condicional o no condicional) en $r_{t}$.

Por tanto, si el conjunto de información disponible es la serie histórica de precios y el modelo de fijación de precios asumido es el paseo aleatorio, la eficiencia débil se identifica con alguno de sus tipos (1, 2 ó 3).

\section{Evaluación de LA EFICIENCIA dÉBIL}

\subsection{MÉTOdOS TRADicionales}

Los procedimientos que tradicionalmente se han utilizado para contrastar la forma débil de eficiencia, tal y como establece alguno de los tipos de paseo aleatorio, se clasifican en dos grupos dependiendo de que utilicen o no para ello técnicas formales de inferencia estadística.

El paseo aleatorio 2 se analiza con métodos que no utilizan técnicas formales de inferencia (regla de los filtros y análisis técnico ${ }^{4}$ ), pues al requerir este tipo de paseo que la serie de rendimientos sea INID, resulta muy complicado contrastar independencia sin asumir distribuciones idénticas (en particular en el contexto de las series temporales), ya que no se podrian obtener las distribuciones muestrales de los estadísticos que se construirian para llevar a cabo el contraste correspondiente.

Por su parte, los procedimientos que aplican técnicas formales de inferencia en el análisis de la eficiencia se pueden clasificar a su vez en dos grupos según permitan contrastar un tipo de paseo aleatorio de forma directa o tan sólo alguna condiciōn necesaria, pero no suficiente, para su cumplimiento.

\footnotetext{
${ }^{4}$ La regla de los filtros y el análisis técnico son dos formas de contrastaciōn empírica de la hipótesis de RW2 que al no utilizar técnicas formales de inferencia estadística se les considera tests "económicos" de la hipótesis de paseo aleatorio 2.
} 
Entre los métodos del segundo grupo encontramos, por un lado, el test de Bartlett, las pruebas basadas en la metodología Box-Jenkins, el test de Box-Pierce, el test de Ljung-Box y el test ratio-varianza, que en realidad solo contrastan la condición de incorrelación de la serie de rendimientos (de ahí que también se les denomine métodos lineales ${ }^{5}$ ) necesaria en cualquiera de los tipos de paseo aleatorio. Ahora bien, como estas pruebas no detectan relaciones no lineales ${ }^{6}$ que, en caso de existir, supondrían la dependencia de la serie, el rechazo de la hipótesis nula implicaría la no incorrelación de la serie $y$, en consecuencia, la inexistencia de cualquier tipo de paseo aleatorio.

Por otro lado, en las pruebas que tratan de detectar efectos $\mathrm{ARCH}$, el rechazo de la hipótesis nula supone únicamente la aceptaciōn de relaciones no lineales, lo cual no implica necesariamente que la serie sea incorrelada.

Frente a los métodos anteriores se encuentran los que permiten determinar directamente si la serie de rendimientos se ajusta a un tipo de paseo aleatorio concreto. Esto significa que dichos procedimientos consideran también la posibilidad de relaciones no lineales en la serie en cuestión, bien porque lo contemple la propia hipótesis nula, o bien porque tengan potencia frente a alternativas que recojan dichas relaciones (serían, pues, métodos no lineales). Entre estos métodos destacan los que permiten contrastar el paseo aleatorio tipo 1 (test BDS, test de rachas y el test de secuencias y reversas) y uno que contrasta el tipo 3 (test ratio-varianza que contempla la heterocedasticidad de la serie).

La clasificaciōn que se ha establecido en esta secciōn para los diferentes métodos que tradicionalmente se utilizan para contrastar la hipótesis de eficiencia débil queda recogida en la Figura 2.

\subsection{Procedimiento aplicado}

Combinando estratégicamente los métodos analizados en el apartado anterior, se aplica un procedimiento para contrastar la hipótesis de paseo aleatorio que se puede iniciar bien desde un método que solo contrasta una condición necesaria pero no suficiente o bien desde uno que contrasta directamente un tipo de paseo $\left(\begin{array}{lll}1 & 0 & 3\end{array}\right)^{7}$.

Por un lado, si al iniciar el procedimiento con un método de los del primer grupo éste pone de manifiesto la incorrelación de la serie de rendimientos, se

\footnotetext{
${ }^{5} \mathrm{~A}$ los procedimientos que tratan de detectar relaciones no lineales se les denomina métodos no lineales con independencia de que sean sensibles o no a la existencia de relaciones lineales.

6 Según Hinich et al. (2005) los sistemas económicos son no lineales y si dicha no linealidad es considerable es un error basarse en una aproximaciōn lineal para hacer predicciones. Es por ello, que estos autores afirman que contrastar la presencia de no linealidad es una forma de validar la linealidad de un sistema.

${ }^{7}$ Puesto que la contrastación de la hipótesis de paseo aleatorio 2 no se basa en la aplicación de técnicas formales de inferencia estadística, en el procedimiento que se propone solo se consideran los tipos 1 y 3.
} 


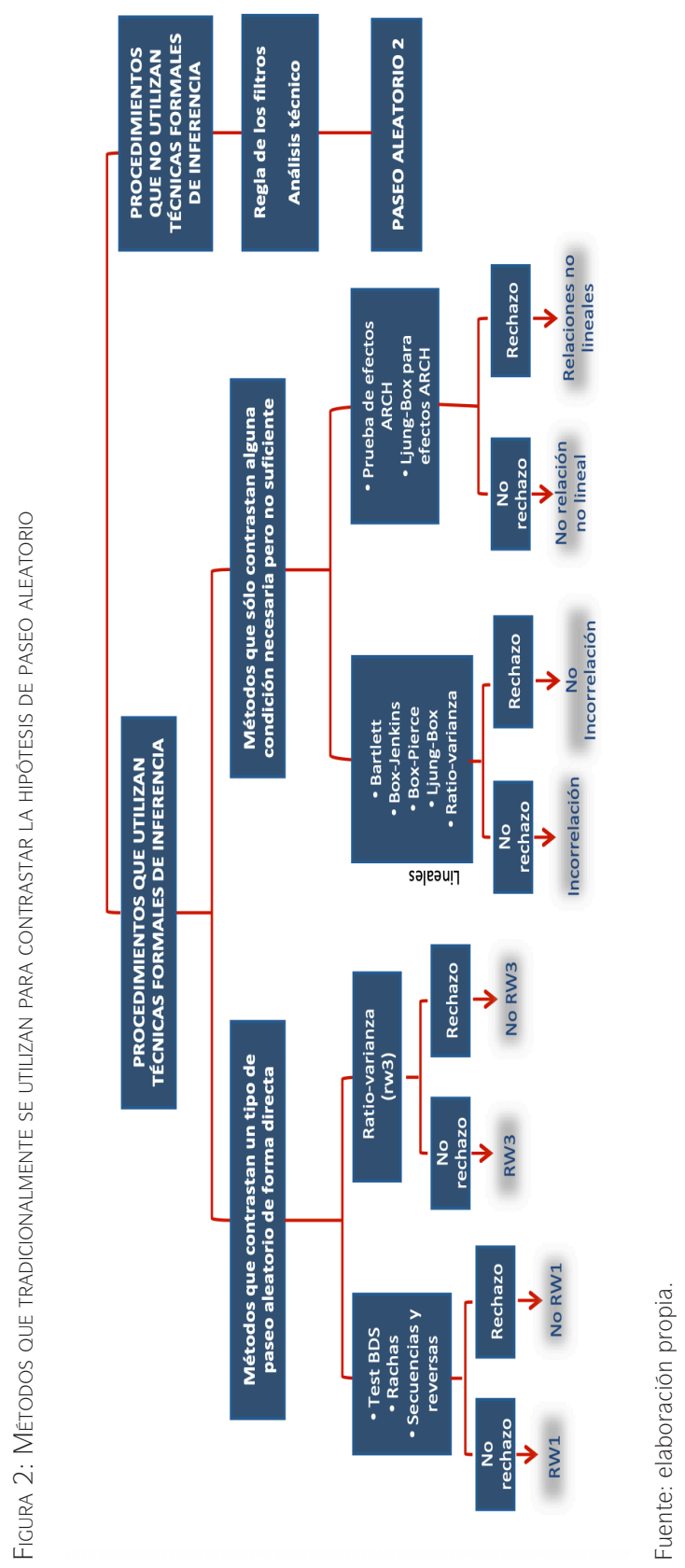


sugiere la aplicación de una prueba de efectos ARCH para determinar qué tipo de paseo es, 1 ó 3. En el caso contrario (correlaciōn), la serie no se ajustaría a ningún tipo de paseo aleatorio.

Por otro lado, en relación con los métodos que contrastan directamente un tipo de paseo aleatorio se propone comenzar el procedimiento con uno que contraste el RW1 de manera que si se rechaza la hipótesis nula con dicho método no se puede descartar que la serie sea RW3 por lo que, antes de afirmar que no estamos ante ningún tipo de paseo aleatorio, se sugiere comprobar este extremo mediante un método que permita su contraste.

El procedimiento descrito se recoge de forma esquemática en la Figura 3.

\subsection{GRAdo DE EFICIENCIA}

Puesto que para una misma serie de rendimientos el procedimiento aplicado puede llevar a conclusiones distintas según cuál sea el test desde el que se inicie, se propone calcular un grado de cumplimiento de la hipótesis de eficiencia débil en el período muestral que resulta de la suma de los valores que se asignan a las conclusiones a las que conduce el procedimiento iniciado desde cada uno de los tests que se consideran para su aplicación. Dichos valores son, el 0 si se rechaza la hipótesis de eficiencia, el 1 si se acepta la eficiencia en la forma que establece el tipo 3 de paseo aleatorio, y el 3 si se acepta en la forma más estricta que establece el paseo aleatorio 1 (obviamente, el valor 2 correspondería al RW2). En el caso de que un determinado test no permita tomar una decisiōn debido a que sus resultados no son concluyentes, se asigna el valor 0,5.

Con esta escala de 0 a 3 se está dando más peso al paseo aleatorio 1 en relación al 3 (en concreto tres veces más) pues, al implicar el RW1 una forma de eficiencia débil más estricta, consideramos que el mercado que la cumpla tiene mayor grado de eficiencia respecto del que solo verifica el tipo 3.

\section{Metodolocía}

La hipótesis de eficiencia débil en los mercados financieros de España, Alemania, Francia e Italia en el período comprendido entre el 1 de enero de 2010 y el 15 de mayo de 2020 se analiza a partir de datos diarios de sus respectivos índices bursátiles (IBEX, DAX, CAC y FTSEMIB) observados en el momento del cierre del mercado correspondiente.

Antes de comprobar la forma débil de la hipótesis de eficiencia en los cuatro mercados indicados, se lleva a cabo un análisis preliminar que permita conocer cuál ha sido el comportamiento de cada uno a lo largo del período considerado, en base a gráficos de evolución de las series de índices de precios 

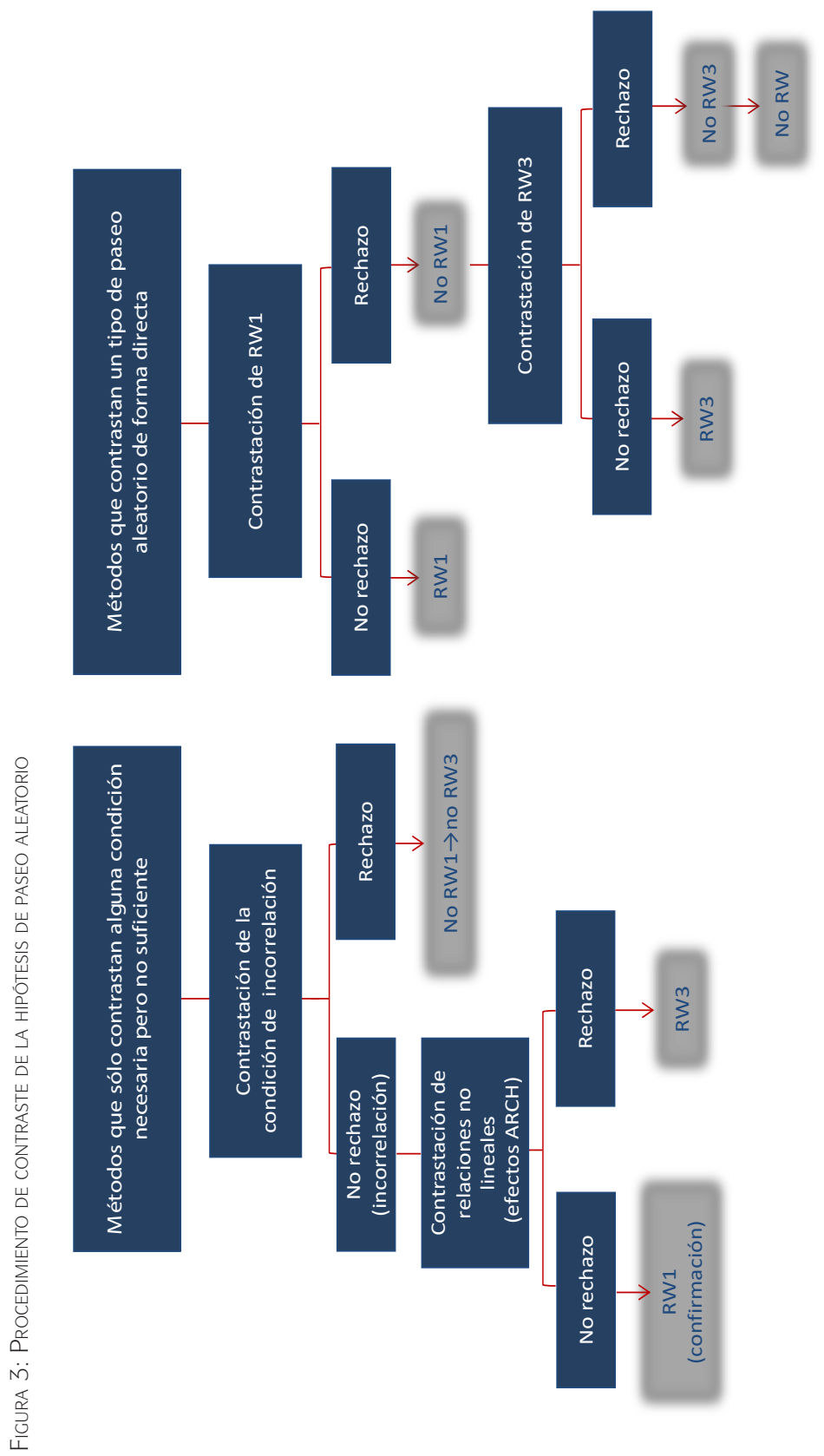

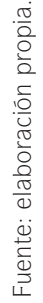


y de los rendimientos asociadas a cada índice, reflejando estos últimos la volatilidad del mercado.

Para determinar si en los diferentes mercados se verifica alguna de las formas de la hipótesis de paseo aleatorio, y con ello la hipótesis de eficiencia débil, se aplica el procedimiento de contraste descrito en la Secciōn 3.2) a las series de rendimientos asociadas a cada índice de precios. En este estudio se consideran el test ratio-varianza propuesto por Lo y MacKinlay (1988) ${ }^{8}$ y el test de Ljung-Box (1978) cuando el procedimiento se inicia desde métodos que sólo contrastan alguna condición necesaria pero no suficiente de la hipótesis de paseo aleatorio, y el test $\operatorname{BDS}^{9}$ y el de rachas (aleatoriedad) cuando se arranca desde métodos que contrastan la citada hipótesis de forma directa. En caso de que el procedimiento requiera la aplicación de una prueba de efectos ARCH para decidir entre los tipos 1 ó 3 de paseo aleatorio, se utilizan modelos ARCH de hasta orden 4.

Finalmente, el grado de cumplimiento de la hipótesis de eficiencia débil en cada mercado se evalúa de acuerdo con la escala definida en la Sección 3.3), esto es, sumando los valores que se asignan a las decisiones a las que conduce el procedimiento iniciado desde diferentes tests. Puesto que en este caso el procedimiento se inicia desde cuatro tests diferentes, el máximo grado de eficiencia se corresponde con el valor 12 (los cuatro tests conducen a RW1) de manera que a medida que nos aproximemos al valor 0 (caso en el que los cuatro tests llevan a rechazar la hipótesis de paseo aleatorio) el grado de eficiencia es cada vez menor.

\section{Resultados}

En esta Sección se presentan los resultados obtenidos tras aplicar la metodología descrita en el apartado anterior a los datos diarios de los índices bursátiles IBEX, DAX, CAC y FTSEMIB correspondientes al período 1 enero de 2010 a 15 de mayo de 2020 .

\footnotetext{
${ }^{8}$ Puesto que el test ratio-varianza que proponen Lo y MacKinlay tiene dos variantes, una que permite contrastar la hipótesis de incorrelación en una serie de rendimientos, y otra que analiza si la citada serie se ajusta a las condiciones de un paseo aleatorio 3, el aplicar una de la dos dependerá de lo que requiera el procedimiento en cada momento. Ahora bien, si el test-ratio-varianza, en cualquiera de sus variantes, conduce a decisiones contradictorias cuando se realiza para diferentes valores del parámetro $k$ (primeros valores de la serie de rendimientos), se decide en base al test global propuesto por Chow y Denning (1993).

${ }^{9}$ Test no paramétrico propuesto por Brock, Dechert, LeBaron y Scheinkman (1996) para contrastar la hipōtesis nula relativa a si una serie es independiente e idénticamente distribuida, que se basa en el concepto de integral de correlación desarrollado por Grassberger y Procaccia (1983) que es una medida de correlación espacial entre dos puntos de un espacio de dimensión $m$ (parámetro de inmersiōn).
} 


\section{1. ANÁLISIS GRÁFICO}

En primer lugar, las representaciones gráficas de los índices analizados y de sus rendimientos asociados, no solo permiten analizar la evoluciōn de cada uno de ellos en el período considerado sino también visualizar los efectos que los diversos acontecimientos acaecidos en dicho período han podido tener en los mercados correspondientes. En concreto, se presta especial atenciōn a los efectos que la declaración de la pandemia del COVID-19 por parte de la Organización Mundial de la Salud (OMS) en marzo de 2020 haya podido provocar en los mercados objeto de análisis, y para facilitar la visualizaciōn de este hecho, se representa la serie de rendimientos de cada índice correspondiente al último año del período observado (mayo 2019 a mayo 2020).

En relaciōn con el mercado español, aunque el índice (IBEX) muestra ciclos de tendencias bajistas y alcistas que se alternan desde 2010 (Figura 4a), el hito clave en el período considerado lo determina la amenaza de Europa de intervenir España, que en 2012 ocasiona que el índice alcance un valor suelo. Precisamente a ese valor suelo se vuelve a aproximar el índice en marzo de 2020, concretamente el día 16, tras la declaraciōn de la pandemia por parte de la OMS. Por otro lado, la representación de la serie de rendimientos del índice (Figura 4b) muestra que

FIGURA 4: INDICE DE PRECIOS Y RENDIMIENTOS ASOCIADOS: IBEX

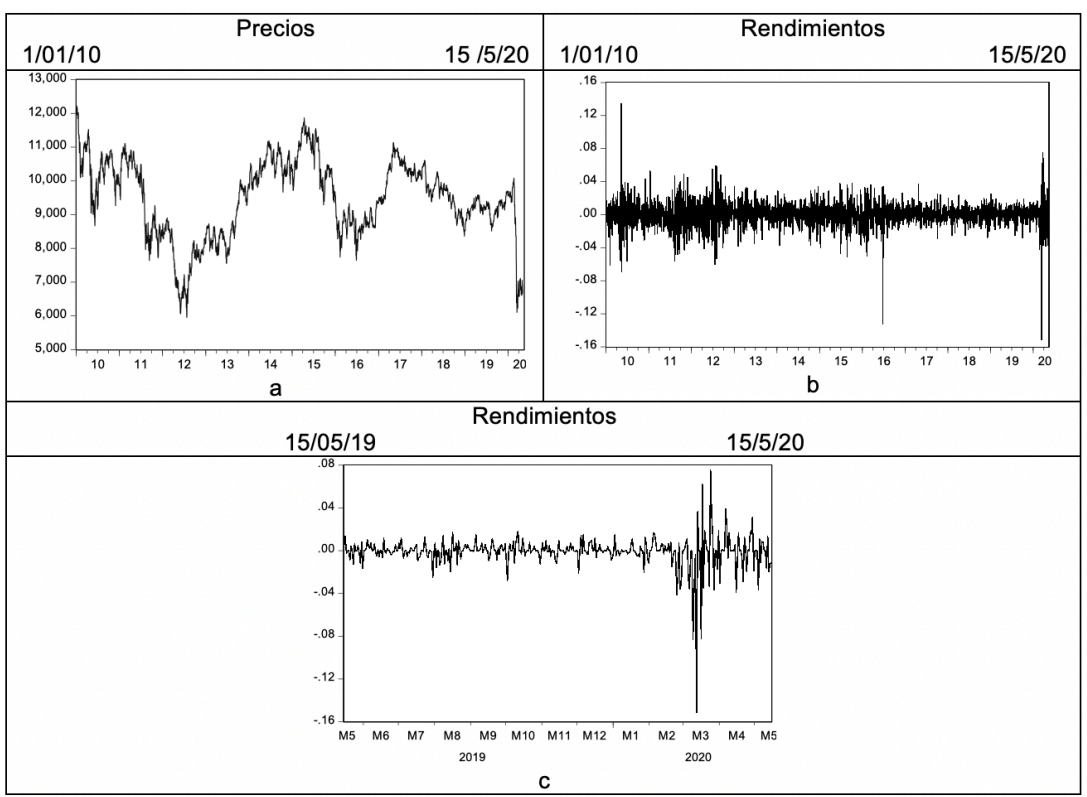

Fuente: elaboraciōn propia. 
los picos más elevados de volatilidad se producen en torno a la declaraciōn de la citada pandemia. Esta circunstancia se aprecia mejor en la Figura 4c, que recoge los rendimientos del IBEX durante el ultimo año (mayo 2019 a mayo 2020). En concreto, se observa claramente un aumento de la volatilidad conforme se acerca el 15 de marzo y, a partir de entonces, la atenuación progresiva de la misma, aunque en ningún momento la disminuciōn lleva a los niveles de comienzos de 2020.

Los datos del mercado francés muestran una tendencia alcista (Figura 5a) a lo largo de los 10 últimos años que se ve interrumpida de forma drástica a inicios de 2020, alcanzando el 18 de marzo un valor suelo a partir del cual comienza una ligera recuperaciōn aún muy lejana de los niveles de precios de finales de 2019. Por su parte, los rendimientos del indice (Figura 5b) muestran que la volatilidad del mercado galo a partir de 2016 es menor que en los años precedentes, comportamiento que sōlo se mantiene hasta 2019. Con el inicio de 2020, la volatilidad empieza a aumentar progresivamente, produciéndose el mayor pico de volatilidad el 12 de marzo, coincidiendo con el rendimiento más bajo del período. Este hecho se puede analizar mejor en la Figura $5 c$ en la que, además, se observa que, a partir del día en el que se produce el

FIGURA 5: INDICE DE PRECIOS Y RENDIMIENTOS ASOCIADOS: CAC

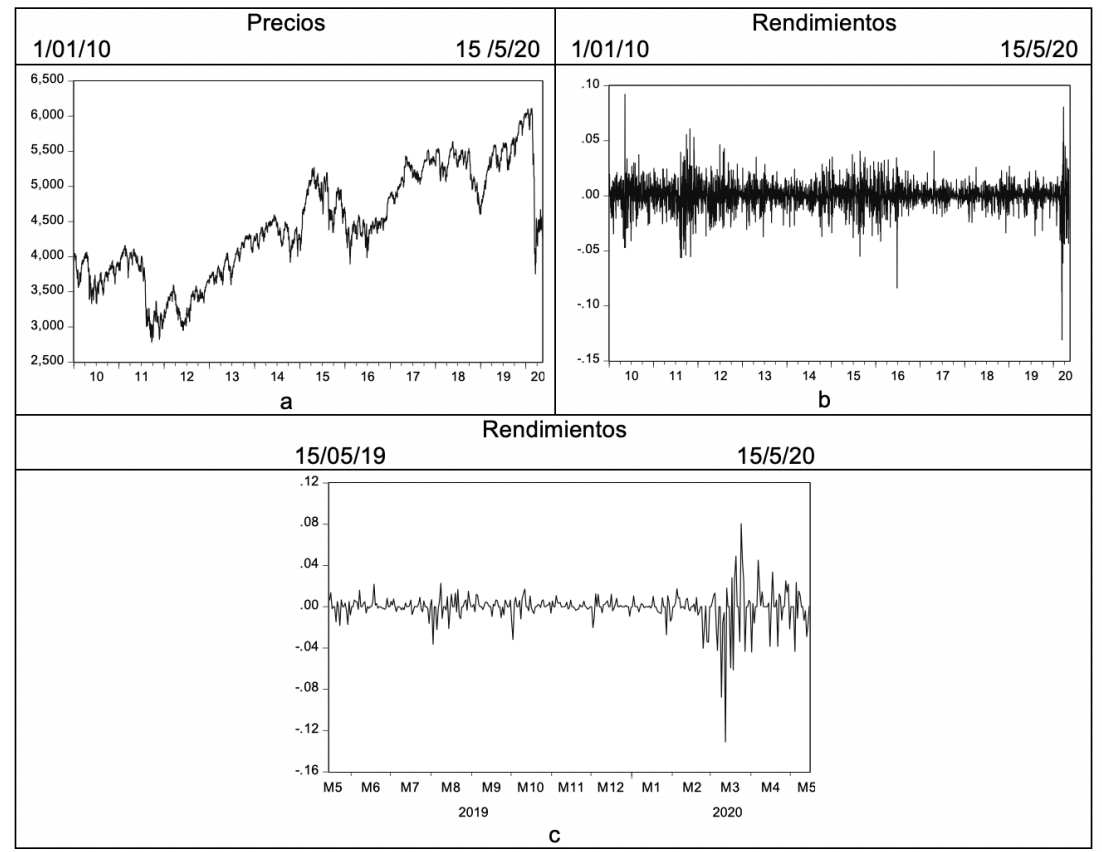

Fuente: elaboración propia. 
rendimiento más elevado (24 de marzo) de este período tan convulso, la volatilidad empieza a disminuir, si bien no llega a niveles tan bajos como los de principios de 2020.

En el mercado alemán, representado por el índice DAX (Figura 6a), se observa una tendencia alcista en los últimos 10 años, durante los cuales, pese a mostrar algunas sub-tendencias bajistas, ningún acontecimiento había ocasionado un descenso tan brusco de este índice como el que se produce con los acontecimientos que preceden a la declaración de la pandemia. En concreto, el citado descenso comienza el 22 de febrero de 2020, alcanzando un valor suelo el 18 de marzo, tras lo cual tan solo llega a recuperar valores próximos a los de 2016. Por otra parte, la representaciōn gráfica de los rendimientos (Figura 6b) pone de manifiesto el comportamiento típico de la volatilidad en series financieras, de modo que se observa como ésta aumenta en los sub-períodos en los que se produce un cambio de tendencia. Especialmente pronunciados son los picos de volatilidad que se producen en marzo de 2020, como refleja con claridad la Figura 6c, en la que se aprecia como la volatilidad aumenta desde final de febrero con picos el 12 y el 24 de

FIGURA 6: INDICE DE PRECIOS Y RENDIMIENTOS ASOCIADOS: DAX

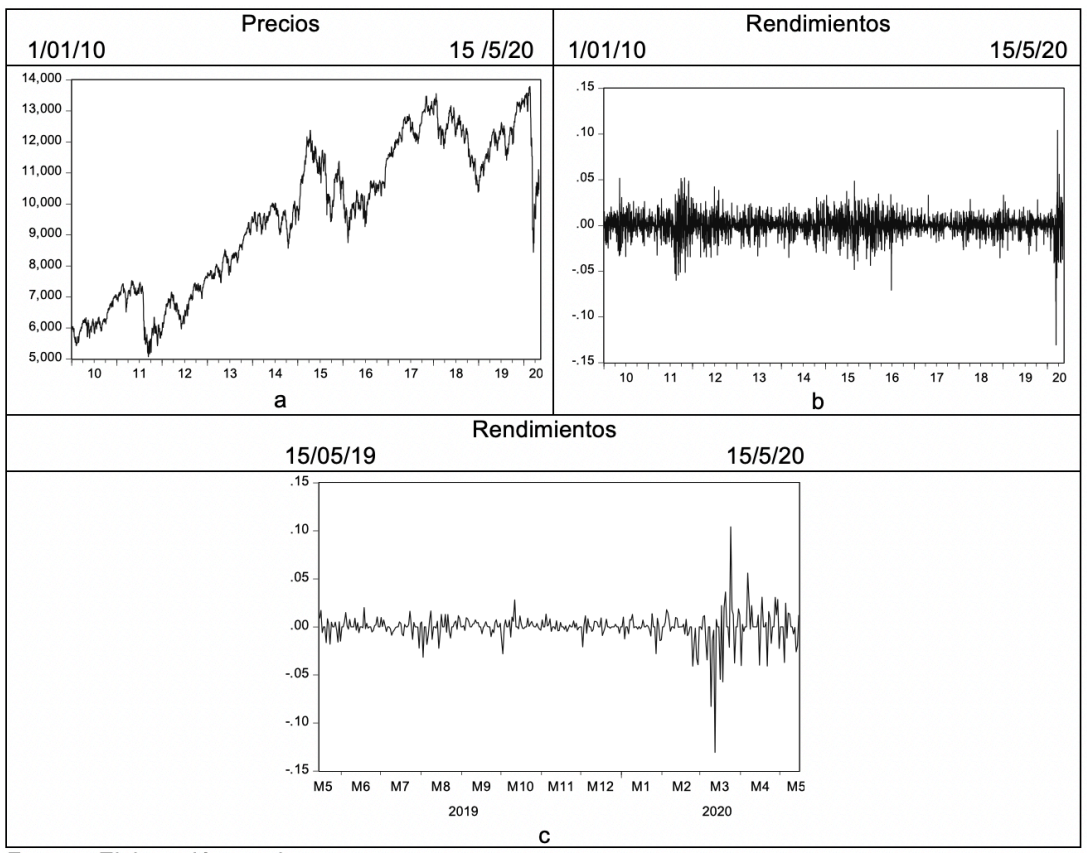

Fuente: elaboraciōn propia. 
marzo, días en los que la rentabilidad alcanzó sus valores mínimo y máximo, respectivamente, en el período muestral considerado. A partir de entonces, se produce un ligero descenso de la volatilidad que no la devuelve aún a niveles precedentes.

El mercado italiano, pese a que su índice (FTSEMIB) muestra ciclos de tendencias alcistas y bajistas que se alternan durante los últimos 10 años (Figura 7a), la declaraciōn de la pandemia ocasiona un comportamiento que no resulta muy novedoso en el período analizado. Tanto es asī, que el descenso del valor que comienza el 19 de febrero de 2020, culmina el 12 de marzo con un valor que aún no se sitúa por debajo del mínimo apreciado en 2016 y, ni mucho menos, del valor suelo de 2012. La representación de los rendimientos (Figura 7b) muestra varios picos de volatilidad ocasionados por valores más elevados de rentabilidad (positiva y negativa) durante el período muestral, si bien los mayores se encuentran en el mes de marzo de 2020, tal como se aprecia de forma más clara en la Figura 7c. Concretamente, el mayor pico de volatilidad se produce el 12 de marzo correspondiente a un valor negativo de rentabilidad que se torna positivo al día siguiente, momento a partir del cual

FIGURA 7: ÍNDICE DE PRECIOS Y RENDIMIENTOS ASOCIADOS: FTSEMIB

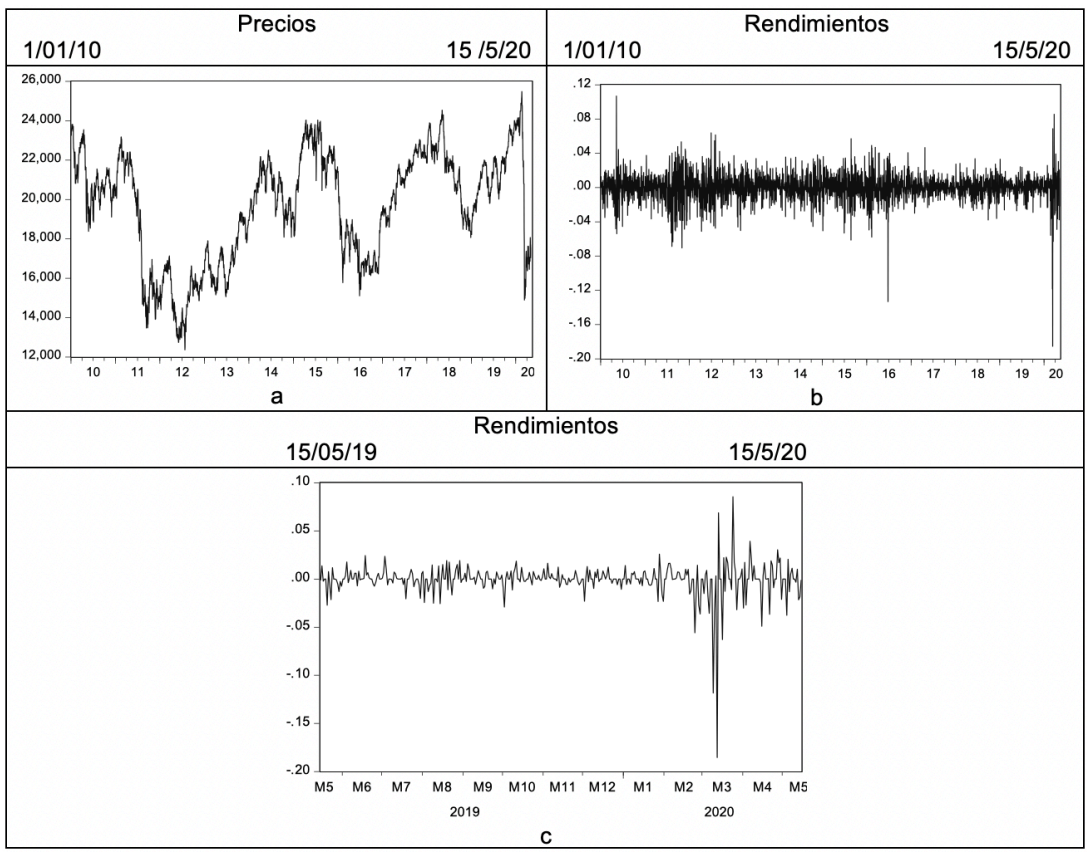

Fuente: elaboraciōn propia. 
se aprecia un descenso paulatino de la volatilidad a medida que van pasando los días.

El hecho de que, en los años objeto de estudio, los mercados español e italiano se mantengan en torno a unos valores constantes (comportamiento típico de mercados laterales) frente a la tendencia alcista que presentan los mercados alemán y francés, se debe, en parte, a la composición sectorial de los propios índices en cada uno de los mercados.

En concreto, en los dos primeros la capitalización relativa por sectores corresponde, en gran medida, al sector servicios financieros e inmobiliarios, uno de los más afectados, no sólo en esta crisis, sino tambiên en la de 2008, de la que aún el IBEX y el FTSEMIB, no se habían recuperado, comportándose aún, como mercados laterales.

En cambio, Alemania y Francia, además de ser dos de los países con mayor peso político y económico en la Unión Europea, destacan por ser mercados cuyos indices están compuestos por importantes empresas del sector industrial y las más representativas en tecnología europea, verdaderos motores de la recuperación.

El comportamiento de los mercados español e italiano no conlleva, necesariamente, que las posiciones de los agentes en dichos mercados sean cortoplacistas, sino que nos encontramos ante mercados laterales fruto de la incertidumbre y de la prima riesgo-paîs asociada. No obstante, las previsiones para los mercados de España e Italia son optimistas. Estos mercados, ahora perjudicados por su carga de empresas del sector bancario, estăn llamados a beneficiarse con la subida generalizada de los índices de precio industriales y de consumo (IPP e IPC), que serán previsiblemente contenidas con la subida de los tipos de interés. Este hecho, unido a la más pronta finalización de la política monetaria acomodaticia por parte de la Uniōn Europea, beneficiará al sector bancario, propiciando que se den posiciones netas de inversión, estratégicas y especulativas, que permitirían que estos mercados abandonasen la lateralidad que muestran.

\subsection{CONTRASTACIŌN DE LA HIPÓTESIS DE EFICIENCIA}

Los resultados de la aplicación del procedimiento descrito en la Sección 3b) sobre cada serie de rendimientos para analizar la hipótesis de eficiencia débil en el correspondiente mercado, se recogen en la Tabla 1. En concreto, dicha tabla detalla, para cada test desde el que se inicie el procedimiento, la combinaciōn de decisiones que lleva a la conclusión final sobre la hipótesis de paseo aleatorio, indicándose, en su caso, el tipo de paseo. Asimismo, los resultados de los tests individuales que requiere la aplicación de cada versión del procedimiento con cada serie de rendimientos para el período considerado se recogen en las Tablas 2 a 7 . 
Asī, comenzando el procedimiento desde el test de Ljung-Box ${ }^{10}$, se concluye ineficiencia en los mercados español e italiano al detectarse correlación en sus respectivas series de rendimientos. Por su parte, la incorrelaciōn que muestran los rendimientos correspondientes a los indices de Alemania y Francia (Tabla 2), nos conduce a corroborar la existencia o no de relaciones no lineales con el fin de determinar la forma en la que se concreta la eficiencia en dichos mercados: paseo aleatorio 1 ó 3. En este sentido, detectados efectos ARCH, es decir, relaciones no lineales en ambas series ( $p$-valor $=0$ en Tabla 4), se concluye la eficiencia de los dos mercados en la forma que establece el paseo aleatorio 3.

Por su parte, el comienzo del procedimiento desde el test ratio-varianza solo conduce a la correlación en el caso del mercado italiano ( $p$-valor $=0,0007$ en Tabla 3) ${ }^{11}$, por lo que se rechaza la eficiencia para este mercado. Para los tres mercados restantes, se acepta la incorrelación de las correspondientes series de rendimientos ( $p$-valor siempre superior a 0,2 en los tests individuales y globales en Tabla 3) que es condiciōn necesaria para la existencia de eficiencia en cualquiera de las formas que establecen los paseos aleatorios 1 ó 3. La detección de efectos ARCH en los rendimientos de los tres mercados ( $p$-valor $=0$ en Tabla 4 ) implica la eficiencia en la forma que establece el paseo aleatorio 3 en España, Alemania y Francia.

El procedimiento iniciado desde el test de rachas evidencia la no aleatoriedad de todas las series de rendimientos analizadas a excepciōn de la correspondiente al FTSEMIB ( $p$-valor $=0,35246$ en Tabla 5). Según estos resultados, solo en el mercado italiano se admitiría la eficiencia en la forma que establece el paseo aleatorio 1, mientras que para el resto, tras la aplicación del test-ratio varianza que contempla la existencia de heterocedasticidad ( $p$-valor superiores a 0,9 en Tabla 7), se concluye que son eficientes conforme a un paseo aleatorio 3.

Resultados similares se obtienen en el caso de iniciar el procedimiento desde el test $B D S^{12}$, que conduce al rechazo de la hipótesis de rendimientos independientes e idénticamente distribuidos en los cuatro mercados analizados ( $p$-valor $=0$ en Tabla 6), descartándose asī el paseo aleatorio $1 \mathrm{y}$, por ende, la eficiencia que éste implica. En cambio, la aplicación del test ratio varianza permite aceptar el paseo aleatorio 3 en todos los mercados ( $p$-valor siempre superior a 0,13 en Tabla 7), lo cual no contradice los resultados del test BDS, pues se admite que los rendimientos son incorrelados pero dependientes.

\footnotetext{
${ }^{10}$ En el contraste de nulidad de los $k$ primeros coeficientes de autocorrelación de cada serie de rendimientos que plantea el test de Ljung-Box, se consideran las 7 primeras autocorrelaciones con la idea de contemplar la posibilidad de correlaciôn semanal (Tabla 2).

${ }^{11}$ Puesto que los diferentes valores de $k$ que se consideran en el test ratio-varianza propuesto por Lo y MacKinlay (1988) conducen a decisiones contradictorias, se utiliza el test global de Chow y Denning (1993) para decidir (Tabla 3).

12 Para el test BDS se ha tomado $m=2,3,4$ y 5 (Tabla 6), pues experimentos Monte Carlo han demostrado que el estadístico de este test presenta buenas propiedades para valores del parámetro de inmersiōn $m$ iguales o menores a 5, con independencia del tamaño muestral (Kanzler, 1999).
} 
TABLA 1: Contrastación de LA HiPŌtesis de EFICIENCIA déBIL: RW1 Ō RW3 FRENTE A NO RW

\begin{tabular}{|c|c|c|c|c|}
\hline $\begin{array}{c}\text { Período } \\
1 / 01 / 10 \text { a } 15 / 05 / 20\end{array}$ & $\begin{array}{c}\text { Ljung-Box } \\
+ \\
\text { (efectos ARCH) }\end{array}$ & $\begin{array}{c}\text { Ratio-Varianza (RW1) } \\
+ \\
\text { (efectos ARCH) }\end{array}$ & $\begin{array}{c}\text { Rachas } \\
+ \\
\text { (ratio-varianza (rw3)) }\end{array}$ & $\begin{array}{c}\text { BDS } \\
+ \\
\text { (ratio-varianza (rw3)) }\end{array}$ \\
\hline $\begin{array}{c}\text { IBEX } \\
\text { (España) }\end{array}$ & $\begin{array}{c}\text { Correlaciôn } \\
\downarrow \\
\text { No RW }\end{array}$ & $\begin{array}{c}\text { Incorrelaciōn } \\
+ \\
\mathrm{ARCH} \\
\downarrow \\
R W 3\end{array}$ & $\begin{array}{c}\text { No aleatoriedad } \\
+ \\
\text { Incorrelación pero } \\
\text { dependencia } \\
\downarrow \\
R W 3\end{array}$ & $\begin{array}{c}\text { No IID } \\
+ \\
\text { Incorrelación pero } \\
\text { dependencia } \\
\downarrow \\
R W 3\end{array}$ \\
\hline $\begin{array}{c}\text { DAX } \\
\text { (Alemania) }\end{array}$ & $\begin{array}{c}\text { Incorrelaciōn } \\
+ \\
\mathrm{ARCH} \\
\downarrow \\
R W 3\end{array}$ & $\begin{array}{c}\text { Incorrelaciōn } \\
+ \\
\mathrm{ARCH} \\
\downarrow \\
R W 3\end{array}$ & $\begin{array}{c}\text { No aleatoriedad } \\
+ \\
\text { Incorrelación pero } \\
\text { dependencia } \\
\downarrow \\
R W 3\end{array}$ & $\begin{array}{c}\text { No IID } \\
+ \\
\text { Incorrelación pero } \\
\text { dependencia } \\
\downarrow \\
R W 3\end{array}$ \\
\hline $\begin{array}{c}\mathrm{CAC} \\
\text { (Francia) }\end{array}$ & $\begin{array}{c}\text { Incorrelaciōn } \\
+ \\
\mathrm{ARCH} \\
\downarrow \\
R W 3\end{array}$ & $\begin{array}{c}\text { Incorrelaciōn } \\
+ \\
\mathrm{ARCH} \\
\downarrow \\
R W 3\end{array}$ & $\begin{array}{c}\text { No aleatoriedad } \\
+ \\
\text { Incorrelación pero } \\
\text { dependencia } \\
\downarrow \\
R W 3\end{array}$ & $\begin{array}{c}\text { No IID } \\
+ \\
\text { Incorrelación pero } \\
\text { dependencia } \\
\downarrow \\
R W 3\end{array}$ \\
\hline $\begin{array}{l}\text { FTSEMIB } \\
\text { (Italia) }\end{array}$ & $\begin{array}{c}\text { Correlaciôn } \\
\downarrow \\
\text { No } R W\end{array}$ & $\begin{array}{c}\text { Correlaciôn } \\
\downarrow \\
\quad \text { No } R W\end{array}$ & $\begin{array}{c}\text { Aleatoriedad } \\
\downarrow \\
R W 1\end{array}$ & $\begin{array}{c}\text { No IID } \\
+ \\
\text { Incorrelación pero } \\
\text { dependencia } \\
\downarrow \\
R W 3\end{array}$ \\
\hline
\end{tabular}

Fuente: elaboraciōn propia.

TABLA 2: TEST DE LJUNG-BoX SOBRE LAS SERIES DE RENDIMIENTOS

\begin{tabular}{|c|c|c|c|c|c|c|c|}
\hline \multirow{3}{*}{$\begin{array}{c}\text { Período } \\
1 / 01 / 10 \text { a } 15 / 05 / 20\end{array}$} & \multicolumn{7}{|c|}{ TEST DE LJUNG-BOX* } \\
\hline & \multicolumn{7}{|c|}{ Orden de correlaciōn (k) } \\
\hline & 1 & 2 & 3 & 4 & 5 & 6 & 7 \\
\hline $\begin{array}{l}\text { IBEX } \\
\text { (España) }\end{array}$ & $\begin{array}{l}0,3902 \\
(0,532)\end{array}$ & $\begin{array}{l}0,3999 \\
(0,819)\end{array}$ & $\begin{array}{l}4,9980 \\
(0,172)\end{array}$ & $\begin{array}{l}8,6286 \\
(0,071)\end{array}$ & $\begin{array}{l}15,412 \\
(0,009)\end{array}$ & $\begin{array}{l}17,758 \\
(0,007)\end{array}$ & $\begin{array}{l}18,503 \\
(0,010)\end{array}$ \\
\hline $\begin{array}{l}\text { DAX } \\
\text { (Alemania) }\end{array}$ & $\begin{array}{l}0,0092 \\
(0,924)\end{array}$ & $\begin{array}{l}0,1457 \\
(0,930)\end{array}$ & $\begin{array}{l}10,379 \\
(0,016)\end{array}$ & $\begin{array}{l}10,556 \\
(0,032)\end{array}$ & $\begin{array}{c}11,121 \\
(0,049)\end{array}$ & $\begin{array}{l}12,281 \\
(0,056)\end{array}$ & $\begin{array}{l}14,173 \\
(0,048)\end{array}$ \\
\hline $\begin{array}{l}\text { CAC } \\
\text { (Francia) }\end{array}$ & $\begin{array}{l}0,3036 \\
(0,582)\end{array}$ & $\begin{array}{l}1,0424 \\
(0,594)\end{array}$ & $\begin{array}{l}4,7005 \\
(0,195)\end{array}$ & $\begin{array}{l}4,8852 \\
(0,299)\end{array}$ & $\begin{array}{l}6,2782 \\
(0,280)\end{array}$ & $\begin{array}{l}6,5960 \\
(0,360)\end{array}$ & $\begin{array}{r}10,111 \\
(0,182)\end{array}$ \\
\hline $\begin{array}{l}\text { FTSEMIB } \\
\text { (Italia) }\end{array}$ & $\begin{array}{l}14,180 \\
(0,000)\end{array}$ & $\begin{array}{l}14,219 \\
(0,001)\end{array}$ & $\begin{array}{l}18,624 \\
(0,000)\end{array}$ & $\begin{array}{l}19,471 \\
(0,001)\end{array}$ & $\begin{array}{l}19,526 \\
(0,002)\end{array}$ & $\begin{array}{l}20,171 \\
(0,003)\end{array}$ & $\begin{array}{l}22,533 \\
(0,002)\end{array}$ \\
\hline
\end{tabular}

* Para cada mercado se recogen los valores del estadístico del test y de su probabilidad límite ( $p$-valor) correspondientes a los diferentes órdenes $k$ que se consideran.

Fuente: elaboración propia. 
TABLA 3: TEST RATIO VARIANZA PARA RW1 SOBRE LAS SERIES DE RENDIMIENTOS

Perî́odo

$1 / 01 / 10$ a $15 / 05 / 20$

\begin{tabular}{|c|c|c|c|c|c|c|c|}
\hline \multirow[b]{2}{*}{$1 / 01 / 10$ a $15 / 05 / 20$} & & & & & & & \\
\hline & $k$ & $V R(k)$ & $k$ & $z$-Statistic & $k$ & $p$-value & Global test** \\
\hline \multirow{4}{*}{$\begin{array}{l}\text { IBEX } \\
\text { (España) }\end{array}$} & 2 & 1,010043 & 2 & 0,618101 & 2 & 0,5365 & \multirow{4}{*}{$\begin{array}{r}1,090432 \\
(0,7245)\end{array}$} \\
\hline & 4 & 1,033146 & 4 & 1,090432 & 4 & 0,2755 & \\
\hline & 8 & 0,982825 & 8 & $-0,357350$ & 8 & 0,7208 & \\
\hline & 16 & 0,948926 & 16 & $-0,714142$ & 16 & 0,4751 & \\
\hline \multirow{4}{*}{$\begin{array}{l}\text { DAX } \\
\text { (Alemania) }\end{array}$} & 2 & 1,001386 & 2 & 0,085294 & 2 & 0,9320 & \multirow{4}{*}{$\begin{array}{r}0,825141 \\
(0,8782)\end{array}$} \\
\hline & 4 & 1,021620 & 4 & 0,711250 & 4 & 0,4769 & \\
\hline & 8 & 1,039658 & 8 & 0,825141 & 8 & 0,4093 & \\
\hline & 16 & 1,017737 & 16 & 0,248011 & 16 & 0,8041 & \\
\hline \multirow{4}{*}{$\begin{array}{l}\text { CAC } \\
\text { (Francia) }\end{array}$} & 2 & 0,991050 & 2 & $-0,550818$ & 2 & 0,5818 & \multirow{4}{*}{$\begin{array}{r}1,238587 \\
(0,6212)\end{array}$} \\
\hline & 4 & 0,986936 & 4 & $-0,429779$ & 4 & 0,6674 & \\
\hline & 8 & 0,964281 & 8 & $-0,743198$ & 8 & 0,4574 & \\
\hline & 16 & 0,911419 & 16 & $-1,238587$ & 16 & 0,2155 & \\
\hline \multirow{4}{*}{$\begin{array}{l}\text { FTSEMIB } \\
\text { (ltalia) }\end{array}$} & 2 & 0,938840 & 2 & $-3,764201$ & 2 & 0,0002 & \multirow{4}{*}{$\begin{array}{r}3,764201 \\
(0,0007)\end{array}$} \\
\hline & 4 & 0,927773 & 4 & $-2,376129$ & 4 & 0,0175 & \\
\hline & 8 & 0,908685 & 8 & $-1,899957$ & 8 & 0,0574 & \\
\hline & 16 & 0,906903 & 16 & $-1,301730$ & 16 & 0,1930 & \\
\hline
\end{tabular}

*En cada mercado, las filas recogen el valor de la razón de varianzas y el del estadístico normalizado $Z$ junto con su probabilidad limite ( $p$-valor), para los diferentes valores de $k$ que se consideran.

**Para cada mercado se recoge el valor del estadístico y probabilidad límite ( $p$-valor) del contraste global de Chow y Denning (1993).

Fuente: elaboración propia.

Tabla 4: Prueba de efectos ARCH sobre las series de Rendimientos

\begin{tabular}{l|r|r|r|r}
\multirow{2}{*}{$\begin{array}{c}\text { Período } \\
\text { 1/01/10 a 15/05/20 }\end{array}$} & \multicolumn{4}{|c}{ PRUEBA DE EFECTOS ARCH* } \\
\cline { 2 - 5 } & \multicolumn{1}{|c}{ Orden de heterocedasticidad $(q)$} \\
\cline { 2 - 5 } & \multicolumn{1}{|c}{2} & \multicolumn{1}{c}{3} & \multicolumn{1}{c}{4} \\
\hline IBEX & 27,54189 & 28,30073 & 91,44510 & 204,0149 \\
(España) & $(0,0000)$ & $(0,0000)$ & $(0,0000)$ & $(0,0000)$ \\
\hline DAX & 11,41629 & 21,35214 & 193,7200 & 232,9889 \\
(Alemania) & $(0,0007)$ & $(0,0000)$ & $(0,0000)$ & $(0,0000)$ \\
\hline CAC & 20,02051 & 34,07435 & 250,7650 & 316,6523 \\
(Francia) & $(0,0000)$ & $(0,0000)$ & $(0,0000)$ & $(0,000)$
\end{tabular}

* Para cada mercado se recogen los valores del estadístico del test y de su probabilidad límite ( $p$-valor) correspondientes a los diferentes órdenes de heterocedasticidad $q$ que se consideran.

Fuente: elaboración propia. 
TABLA 5: TeSt de RACHAS (ALEATORIEDAD) SOBRE LAS SERIES DE RENDIMIENTOS

\begin{tabular}{|c|c|c|}
\hline \multirow{2}{*}{$\begin{array}{c}\text { Período } \\
1 / 01 / 10 \text { a } 15 / 05 / 20\end{array}$} & \multicolumn{2}{|c|}{ Runs test } \\
\hline & Número de rachas* & $p$-valor \\
\hline $\begin{array}{l}\text { IBEX } \\
\text { (España) }\end{array}$ & 1562 & $1,43 \cdot 10^{-6}$ \\
\hline $\begin{array}{l}\text { DAX } \\
\text { (Alemania) }\end{array}$ & 1644 & $3,74 \cdot 10^{-5}$ \\
\hline $\begin{array}{l}\text { CAC } \\
\text { (Francia) }\end{array}$ & 1638 & $5,94 \cdot 10^{-5}$ \\
\hline $\begin{array}{l}\text { FTSEMIB } \\
\text { (Italia) }\end{array}$ & 1660 & 0,35246 \\
\hline
\end{tabular}

* Se toma la media muestral de cada serie para establecer las rachas

Fuente: elaboración propia.

TABLA 6: TEST BDS SOBRE LAS SERIES DE RENDIMIENTOS

\begin{tabular}{|c|c|c|c|c|c|c|}
\hline \multirow{2}{*}{$\begin{array}{c}\text { Período } \\
1 / 01 / 10 \text { a } 15 / 05 / 20\end{array}$} & \multicolumn{6}{|c|}{ BDS TEST* } \\
\hline & m & BDS Statistic & $m$ & $z$-Statistic & $m$ & $p$-value \\
\hline \multirow{4}{*}{$\begin{array}{l}\text { IBEX } \\
\text { (España) }\end{array}$} & 2 & 0,019661 & 2 & 9,798556 & 2 & 0,0000 \\
\hline & 3 & 0,017368 & 3 & 7,220143 & 3 & 0,0000 \\
\hline & 4 & 0,013164 & 4 & 6,077897 & 4 & 0,0000 \\
\hline & 5 & 0,010212 & 5 & 5,975390 & 5 & 0,0000 \\
\hline \multirow{4}{*}{$\begin{array}{l}\text { DAX } \\
\text { (Alemania) }\end{array}$} & 2 & 0,019255 & 2 & 9,277426 & 2 & 0,0000 \\
\hline & 3 & 0,016626 & 3 & 6,635379 & 3 & 0,0000 \\
\hline & 4 & 0,013335 & 4 & 5,868726 & 4 & 0,0000 \\
\hline & 5 & 0,010420 & 5 & 5,770366 & 5 & 0,0000 \\
\hline \multirow{4}{*}{$\begin{array}{l}\text { CAC } \\
\text { (Francia) }\end{array}$} & 2 & 0,023972 & 2 & 11,67477 & 2 & 0,0000 \\
\hline & 3 & 0,023606 & 3 & 9,464000 & 3 & 0,0000 \\
\hline & 4 & 0,020368 & 4 & 8,949953 & 4 & 0,0000 \\
\hline & 5 & 0,017083 & 5 & 9,387889 & 5 & 0,0000 \\
\hline \multirow{4}{*}{$\begin{array}{l}\text { FTSEMIB } \\
\text { (Italia) }\end{array}$} & 2 & 0,018236 & 2 & 9,272420 & 2 & 0,0000 \\
\hline & 3 & 0,015304 & 3 & 6,633624 & 3 & 0,0000 \\
\hline & 4 & 0,011628 & 4 & 5,720286 & 4 & 0,0000 \\
\hline & 5 & 0,008176 & 5 & 5,208593 & 5 & 0,0000 \\
\hline
\end{tabular}

*En cada mercado, las filas recogen el valor del estadístico BDS y el del estadīstico normalizado $Z$ junto con su probabilidad límite ( $p$-valor), para los diferentes valores del parămetro de inmersiōn $m$ que se consideran.

Fuente: elaboraciōn propia. 
TABLA 7: TEST RATIO VARIANZA PARA RW3 SOBRE LAS SERIES DE RENDIMIENTOS

\begin{tabular}{|c|c|c|c|c|c|c|c|}
\hline \multirow{2}{*}{$\begin{array}{c}\text { Período } \\
1 / 01 / 10 \text { a } 15 / 05 / 20\end{array}$} & \multicolumn{7}{|c|}{ TEST RATIO VARIANZA (RW3)* } \\
\hline & k & $\mathrm{VR}(k)$ & k & $z$-Statistic & $k$ & $p$-value & $\begin{array}{l}\text { Global } \\
\text { test** }\end{array}$ \\
\hline \multirow{4}{*}{$\begin{array}{l}\text { IBEX } \\
\text { (España) }\end{array}$} & 2 & 1,010310 & 2 & 0,385682 & 2 & 0,6997 & \multirow{4}{*}{$\begin{array}{r}0,720002 \\
(0,9220)\end{array}$} \\
\hline & 4 & 1,033965 & 4 & 0,720002 & 4 & 0,4715 & \\
\hline & 8 & 0,984645 & 8 & $-0,191332$ & 8 & 0,8483 & \\
\hline & 16 & 0,952700 & 16 & $-0,393094$ & 16 & 0,6943 & \\
\hline \multirow{4}{*}{$\begin{array}{l}\text { DAX } \\
\text { (Alemania) }\end{array}$} & 2 & 1,001650 & 2 & 0,074850 & 2 & 0,9403 & \multirow{4}{*}{$\begin{array}{r}0,551047 \\
(0,9694)\end{array}$} \\
\hline & 4 & 1,022430 & 4 & 0,519572 & 4 & 0,6034 & \\
\hline & 8 & 1,041583 & 8 & 0,551047 & 8 & 0,5816 & \\
\hline & 16 & 1,021785 & 16 & 0,186823 & 16 & 0,8518 & \\
\hline \multirow{4}{*}{$\begin{array}{l}\text { CAC } \\
\text { (Francia) }\end{array}$} & 2 & 0,991312 & 2 & $-0,368452$ & 2 & 0,7125 & \multirow{4}{*}{$\begin{array}{r}0,696101 \\
(0,9304)\end{array}$} \\
\hline & 4 & 0,987719 & 4 & $-0,268380$ & 4 & 0,7884 & \\
\hline & 8 & 0,966066 & 8 & $-0,424762$ & 8 & 0,6710 & \\
\hline & 16 & 0,915043 & 16 & $-0,696101$ & 16 & 0,4864 & \\
\hline \multirow{4}{*}{$\begin{array}{l}\text { FTSEMIB } \\
\text { (ltalia) }\end{array}$} & 2 & 0,939088 & 2 & $-2,113749$ & 2 & 0,0345 & \multirow{4}{*}{$\begin{array}{r}2,113749 \\
(0,1312)\end{array}$} \\
\hline & 4 & 0,928509 & 4 & $-1,394816$ & 4 & 0,1631 & \\
\hline & 8 & 0,910368 & 8 & $-1,068320$ & 8 & 0,2854 & \\
\hline & 16 & 0,910509 & 16 & $-0,756458$ & 16 & 0,4494 & \\
\hline
\end{tabular}

*En cada mercado, las filas recogen el valor de la razón de varianzas y el del estadístico normalizado $Z$ junto con su probabilidad limite ( $p$-valor), para los diferentes valores de $k$ que se consideran.

**Para cada mercado se recoge el valor del estadístico y probabilidad límite ( $p$-valor) del contraste global de Chow y Denning (1993).

Fuente: elaboraciōn propia.

\subsection{GRAdo de EFICIENCIA}

Finalmente, en la Tabla 8 se muestran los resultados que permiten analizar el grado de cumplimiento de la hipótesis de eficiencia débil en cada mercado en la forma indicada en la metodología. En concreto, cada columna de la tabla recoge, para cada índice, los valores que, según la escala definida en la Secciōn 3c), se asignan a la decisiōn a la que conduce el procedimiento iniciado desde cada uno de los cuatro tests considerados, así como la suma de dichos valores. Esta suma supone una aproximaciōn cuantitativa al grado de eficiencia alcanzado en cada uno de los mercados.

De los resultados obtenidos destaca la homogeneidad detectada en los mercados alemán y francés, pues en los dos casos el procedimiento conduce a la aceptación de la eficiencia en la forma que establece el paseo aleatorio 3 independientemente del test desde el cual se inicie dicho procedimiento. Ambos mercados alcanzan un grado de eficiencia total igual a 4 (máximo posible cuando se logra la eficiencia en la forma que establece el paseo aleatorio 3 en todos los casos).

Por su parte, el mercado italiano, pese a tener el mismo grado de eficiencia que Alemania y Francia, presenta datos menos homogéneos. En concreto, el procedimiento detecta ineficiencia cuando se inicia desde métodos que 
contrastan solo una condición necesaria pero no suficiente para aceptar cualquier tipo de paseo aleatorio, mientras que cuando arranca desde métodos que contrastan de forma directa un tipo de paseo, en un caso lleva al paseo aleatorio 1 (iniciando desde el test BDS) y en otro al tipo 3 (comenzando desde el test de rachas).

Por último, el mercado que menor grado de eficiencia alcanza es el español, pues en tres versiones del procedimiento tan solo se detecta la forma de la eficiencia que establece el paseo aleatorio 3, obteniéndose así un grado de eficiencia total igual a 3.

El que se detecten grados de eficiencia diferentes en el contexto de integraciōn que supone la Uniōn Europea, puede justificarse por las peculiaridades de la economía de cada país y, en particular, por las diferencias existentes en el peso que tienen los distintos sectores económicos que componen el índice de precios correspondiente.

TABLA 8: GRAdo de CUMPLIMIENTO de LA HiPótesis de EFICIENCIA dÉBIL (RW1 Ó RW3)

\begin{tabular}{|c|c|c|c|c|}
\hline $\begin{array}{c}\text { Período } \\
1 / 01 / 10 \text { a } 15 / 05 / 20\end{array}$ & $\begin{array}{c}\text { IBEX } \\
\text { (España) }\end{array}$ & $\begin{array}{c}\text { DAX } \\
\text { (Alemania) }\end{array}$ & $\begin{array}{c}\text { CAC } \\
\text { (Francia) }\end{array}$ & $\begin{array}{l}\text { FTSEMIB } \\
\text { (Italia) }\end{array}$ \\
\hline $\begin{array}{l}\text { LB } \\
+ \\
\text { (ARCH) }\end{array}$ & 0 & 1 & 1 & 0 \\
\hline $\begin{array}{l}\text { R-V (rw1) } \\
+ \\
\text { (ARCH) }\end{array}$ & 1 & 1 & 1 & 0 \\
\hline $\begin{array}{l}\text { Rachas } \\
+ \\
\text { (R-V (rw3)) }\end{array}$ & 1 & 1 & 1 & 3 \\
\hline $\begin{array}{l}\text { BDS } \\
+ \\
(\mathrm{R}-\mathrm{V}(\mathrm{rw} 3))\end{array}$ & 1 & 1 & 1 & 1 \\
\hline TOTAL* & 3 & 4 & 4 & 4 \\
\hline
\end{tabular}

*Valor mínimo $=0 \rightarrow$ las 4 versiones del procedimiento conducen a ineficiencia.

Valor máximo $=12 \rightarrow$ las 4 versiones del procedimiento conducen a eficiencia en su forma más estricta: RW1.

Fuente: elaboraciōn propia.

\section{CONCLUSIONES}

El objetivo principal de este trabajo era analizar la forma débil de la eficiencia desde la perspectiva de los tipos de paseo aleatorio (1, 2 y 3) en cuatro mercados financieros europeos (España, Alemania, Francia e Italia) en el período 1 de enero de 2010 a 15 de mayo de 2020.

En un estudio preliminar, el análisis gráfico de la evolución de los índices objeto de estudio permite concluir que a lo largo del periodo considerado la tendencia general en los mercados de Alemania y Francia ha sido alcista, 
mientras que en España e Italia dicha tendencia se ha mantenido constante. No obstante, las series de rendimientos asociadas al índice de precios de cada mercado ponen de manifiesto que, en los cuatro mercados, la mayor volatilidad en el período considerado se produce tras la declaración de la pandemia del COVID-19 por parte de OMS, es decir, a mediados de marzo de 2020.

El procedimiento que, combinando métodos que contrastan solo una condición necesaria pero no suficiente para el cumplimiento de la hipótesis de paseo aleatorio y métodos que contrastan directamente un tipo de paseo aleatorio concreto, permite detectar si una serie de rendimientos asociados a un índice de precios sigue un paseo aleatorio, indica que, en general, los cuatro mercados analizados son eficientes en la forma que establece el paseo aleatorio 3. Como se ha comentado en este trabajo, la versiōn 3 del paseo aleatorio es más flexible que los tipos 1 y 2 en cuanto a las condiciones que se requieren para su cumplimiento (incorrelaciōn, pero dependencia), pues contemplan la posibilidad de relaciones no lineales en la serie de rendimientos, en cuyo caso sería factible plantear, por ejemplo, modelos de volatilidad.

Los mercados que presentan mayor grado de cumplimiento de la hipótesis de eficiencia débil de acuerdo con la escala de valoración propuesta en este trabajo son el alemán, el francés y el italiano. Si bien en los casos de Alemania y Francia el grado de eficiencia viene determinado claramente por el paseo aleatorio tipo 3, el resultado del caso de Italia no muestra la homogeneidad de los dos primeros. Finalmente, el mercado español es el que presenta menor grado de eficiencia.

Puesto que uno de los objetivos era estudiar el impacto inicial de la crisis del COVID-19 en la eficiencia de los cuatro mercados analizados y el cumplimiento de la eficiencia en los mercados financieros suele tener un carácter transitorio, el período muestral considerado finaliza poco después de la declaración de la pandemia (15 de mayo de 2020). Se llegó a estudiar, incluso, la eficiencia antes de marzo de 2020 y los resultados coincidian con los obtenidos en este trabajo. En la actualidad, los autores están analizando la incidencia del COVID-19 en la eficiencia de los mercados desde una perspectiva más a largo plazo con series de rendimientos que comprenden un período de tiempo más amplio desde el inicio de la pandemia.

\section{REFERENCIAS}

Bachelier, L. (1900). Théorie de la Spéculation. In Annales Scientifiques de I E.N.S., 3e série, tome 17, 21-86.

Bailey, R. E. (2005). The Economics of Financial Markets. Cambridge University Press, New York.

Brock, W. A., Decher, W. D. and Scheinkman, J. A. (1987). A Test for Independence Based on the Correlation Dimension. University of Wisconsin at Madison, Department of Economics Working Paper. 
Brock, W. A., Decher, Lebaron, B. and W. D. and Scheinkman, J. A. (1996). A Test for Independence Based on the Correlation Dimension. Econometric Reviews, 15(3), 197-235.

Campbell, J. Y., Lo, A. W. and Mackinlay, A. C. (1997). The Econometrics of Financial Markets. Princeton University Press, New Jersey.

Chow, K.V. and Denning, K.C. (1993). A Simple Multiple Variance Ratio Test. Journal of Econometrics, 58(3), 385-401.

Fama, E. F. (1970). Efficient Capital Markets: A Review of Theory and Empirical Work. The Journal of Finance, 25(2), 383-417.

Fama, E. F. (1991). Efficient Capital Markets: II. The Journal of Finance, 46(5), 1575-1617.

Grassberger, P. and Procaccia, I. (1983). Characterization of Strange Attractors. Physical Review Letters, 50(5), 346-349.

Hinich, M. J., Mendes, E. M. and Stone. L. (2005). A Comparison between Standard Bootstrap and Theiler's Surrogate Methods, University of Texas, Austin.

Kanzler, L. (1999). Very Fast and Correctly Sized Estimation of the BDS Statistic. Unpublished manuscript. Department of Economics, University of Oxford.

Ljung, G. M. and Box, G. E. P. (1978). On a Measure of lack of Fit in Time Series Models. Biometrika, 65(2), 297-303.

Lo, A. W. and MacKinlay, A. C. (1988). Stock Market Prices do not Follow Random Walks: Evidence from a Simple Specification Test. The Review of Financial Studies, 1(1), 41-66.

Roberts, H. V. (1967). Statistical versus Clinical Prediction of the Stock Market. Unpublished paper presented at The Seminar on the Analysis of the Security Prices, University of Chicago.

Samuelson, P. A. (1965). Proof that Properly Anticipated Prices Fluctuate Randomly. Industrial Management Review, 6(2), 41-49. 
